\title{
Les officiers du présidial d'Angers dans la seconde moitié du XVIIIe siècle : étude sociale
}

The officers of the présidial of Angers in the second half of the eighteenth century: a social study

Jacques Maillard

\section{(2) OpenEdition}

\section{Journals}

Édition électronique

URL : http://journals.openedition.org/abpo/2574

DOI : $10.4000 /$ abpo.2574

ISBN : 978-2-7535-2782-9

ISSN : 2108-6443

\section{Éditeur}

Presses universitaires de Rennes

\section{Édition imprimée}

Date de publication : 30 mars 2013

Pagination : 153-162

ISBN : $978-2-7535-2780-5$

ISSN : 0399-0826

Référence électronique

Jacques Maillard, «Les officiers du présidial d'Angers dans la seconde moitié du XVIIle siècle : étude sociale », Annales de Bretagne et des Pays de l'Ouest [En ligne], 120-1 | 2013, mis en ligne le 30 mars 2015, consulté le 01 mai 2019. URL : http://journals.openedition.org/abpo/2574 ; DOI : 10.4000/ abpo. 2574 


\title{
Les officiers du présidial d'Angers dans la seconde moitié du XVIII ${ }^{\mathrm{e}}$ siècle : étude sociale
}

\author{
Jacques MAILLARD \\ professeur émérite à l'université d'Angers \\ CERHIO UMR 6258-université d'Angers
}

Le présidial d'Angers ${ }^{1}$ est la plus importante des institutions judiciaires de la province d'Anjou ${ }^{2}$. Créé en 1552 par Henri II, il a très vite attiré en son sein les membres des grandes familles angevines, présentes aussi à l'échevinage d'Angers et au chapitre de la cathédrale. Pendant de nombreuses années, les officiers du présidial ont accaparé les places dans ces deux grandes institutions angevines. Le point culminant de cette domination peut être situé dans la seconde moitié $\mathrm{du}_{\mathrm{XVII}}^{\mathrm{e}}$ siècle. Fidèles au roi lors des troubles de la Fronde qui ont agité l'Anjou, les officiers du présidial ont obtenu, verbalement ${ }^{3}$, le droit de porter la robe rouge dans toutes les cérémonies publiques. Les maires d'Angers et les échevins, nommés alors par le roi puis par le gouverneur de la province, car la ville a perdu le droit de désigner ses édiles librement, sont choisis en son sein, comme les capitaines des compagnies de la milice bourgeoise. Toute autre apparaît la situation après 1750, période de déclin de l'institution angevine. Nous nous proposons de présenter les principaux aspects de ce déclin avant de nous interroger sur les conséquences de celui-ci pour les hommes chargés de rendre la justice dans la capitale de la province, conséquences qui apparaissent dans un nouveau recrutement familial et dans une transformation du mode de vie des officiers de cette institution.

1. Ce texte reprend et développe la communication présentée lors des Journées régionales d'histoire de la justice, qui se sont tenues à Angers les 23 et 24 novembre 2007.

2. Cet article doit beaucoup à l'excellent travail d'un de mes étudiants, AzEMA, Christophe, Les juges magistrats du présidial dans la seconde moitié du XVIII siècle. Fortune et famille, mémoire de maîtrise, université d'Angers, 1996.

3 . En mai 1683 ils obtiennent des lettres patentes de confirmation, Bibliothèque nationale de France, ms. Joly de Fleury, 2161. 


\section{Les aspects du déclin du présidial}

Les aspects de ce déclin sont nombreux et d'importance variable ${ }^{4}$. Cinq nous paraissent essentiels.

La perte d'influence au sein de l'échevinage d'Angers, tout d'abord. De 1657 à 1789, les officiers du présidial ont fourni $16 \%$ des membres de l'assemblée municipale, dont plus de $40 \%$ des maires. Mais ces chiffres cachent une évolution très nette caractérisée par la diminution de la place qu'ils y occupent. En 1660, ils représentaient plus de $33 \%$ des élus, ils sont moins de $6 \%$ à la veille de la Révolution. Le déclin est moins marqué, toutefois, pour la fonction de maire, le présidial fournissant encore $25 \%$ des maires élus de 1773 à $1789^{5}$.

La perte d'influence du présidial au sein de la milice bourgeoise de la ville est sans doute une conséquence de la diminution du nombre des officiers royaux au sein de l'assemblée municipale. Depuis la création de la mairie d'Angers par Louis XI en 1475, le maire porte le titre de " capitaine général de la ville " et il a le droit de mettre les habitants sous les armes pour " faire guet et garde " aux portes de la ville. Depuis 1560, la sécurité d'Angers est assurée par une milice bourgeoise qui reste très active jusqu'à la Révolution. Les capitaines des compagnies de cette milice ${ }^{6}$ sont nommés par l'échevinage. Les officiers du présidial représentent $62 \%$ des 23 capitaines désignés en 1660 . À cette date, le présidial qui est maître de l'échevinage possède également le commandement de la milice bourgeoise. De 1770 à 1789, 27 capitaines ont été désignés : aucun n'appartient au présidial. Plus de $57 \%$ de ces capitaines sont des marchands. Ceux-ci accèdent alors en grand nombre à l'échevinage et s'emparent de ces charges de capitaines.

La perte de la responsabilité de la police de la ville est une autre marque du déclin de la compagnie présidiale angevine. En 1699, la création d'un lieutenant général de police a transformé l'organisation d'une police alors mal assurée par les officiers de la prévôté royale. Échevinage et présidial se disputent la maîtrise de cette nouvelle institution. Si le corps de ville réussit à obtenir, en 1709, la réunion à l'échevinage des deux conseillers de police chargés d'assister le lieutenant général de police, il échoue dans toutes ses tentatives pour dominer l'institution et ne peut empêcher en 1734 le présidial d'obtenir la réunion de l'office de Lieutenant général de police dont il a fait l'acquisition ${ }^{7}$. Mais, en 1762, à la mort du conseiller Boguais, sous le

4. Sur ce déclin qui se rencontre dans de nombreux tribunaux, voir GRESSET, Maurice, Gens de justice à Besançon de la conquête par Louis XIV à la Révolution (1674-1789), 2 vol., Paris 1975 et TOURNERIE, Jean-André, Recherches sur la crise judiciaire en province à la fin de l'Ancien Régime, mémoire de maîtrise, Tours, 1975, 126 pages.

5. Sur cette évolution, voir MAILLARD, Jacques, Le Pouvoir municipal à Angers de 1657 à 1789, Angers, 1984, t.1, p. 227-232.

6. Le nombre des compagnies a varié à plusieurs reprises. En 1657, ce nombre est porté de 12 à 23. En 1770 il est à nouveau fixé à 12, MAILlARD, Jacques, Le Pouvoir municipal..., op. cit., t. 2, p. 80-91.

7. En janvier 1730, à la mort d'Audouin de la Blanchardière " président de la prévôté, juge civil et criminel ", le corps de ville réussit à acheter l'office à ses héritiers, mais le 
nom duquel le présidial avait obtenu les provisions de l'office, ce dernier doit être levé aux parties casuelles, faute de paiement du droit annuel. Les officiers du présidial sont incapables de verser les 44000 livres demandées. Si en 1771 ils lèvent enfin cet office, c'est pour le revendre aussitôt à JeanFrançois Allard, conseiller de l'hôtel de ville, pour 48000 livres, et ce dernier doit pour ce paiement rembourser de nombreux emprunts à la place des officiers de l'institution judiciaire ${ }^{8}$. Quand, en 1780, Allard revend son office au corps de ville, les officiers du présidial sont incapables de s'y opposer et, après quelques années de conflits, doivent accepter que l'office acheté par l'échevinage soit réuni à ce dernier. Le 10 mars 1783, ils sont obligés d'accorder " entrée, séance et voix délibérative " au nouveau Lieutenant général de police, après avoir obtenu que la charge ne puisse être exercée que par le maire, espérant ainsi ne pas avoir à recevoir un marchand en leur sein ${ }^{9}$. Vaine précaution, car, en 1785 , le nouveau maire, Charles-Félix Claveau est négociant " en vin et en ardoises ". Dans les années qui précèdent la Révolution, le corps de ville contrôle donc entièrement la police à Angers. Le 18 février 1783 l'échevinage a décidé d'exercer les fonctions de police gratuitement, "il ne sera payé aucune épices ny vacation pour le juge dans toutes les affaires de voiries et de police quelles conques, même de parties à parties ${ }^{10}$ ". Le nombre des affaires portées devant le tribunal de police augmente rapidement ${ }^{11}$.

En 1762, si le présidial a été incapable de payer les sommes demandées pour lever l'office de Lieutenant général de police aux parties casuelles, c'est parce qu'il est, alors très endetté. La situation financière de la compagnie n'a cessé de se dégrader depuis 1740 comme le montrent les problèmes rencontrés lors de la suppression de la prévôté. Les officiers du présidial avaient à plusieurs reprises demandé au roi la réunion de ce tribunal considéré comme inutile ${ }^{12}$, mais lorsqu'en juillet 1746 le chancelier leur demande s'ils étaient " en état et disposition " de contribuer au remboursement des offices qui seraient supprimés, ils répondent que le projet de suppression de la prévôté est " avantageux au bien public ", mais que l'état financier de la compagnie rend impossible l'opération ${ }^{13}$. Or, en 1749 , la prévôté royale a été supprimée et ses offices ont été unis au présidial. Une partie des

présidial fait échouer l'opération en intervenant auprès du roi qui refuse d'accorder à la ville les moyens financiers nécessaires à l'opération.

8. Arch. dép. de Maine-et-Loire, 5 E 1/1076, notaire Deville.

9. BnF, ms. Joly de Fleury $385, \mathrm{f}^{\circ} 68$.

10. Arch. mun. d'Angers, BB 130, f 80.

11. Les officiers du présidial considèrent que la suppression de la prévôté, en 1749, n'a pas simplifié l'organisation judiciaire et n'a pas entraîné une augmentation du nombre des affaires portées devant eux car les habitants d'Angers et de la province préfèrent utiliser les services de tribunaux plus proches d'eux, comme les justices seigneuriales et le tribunal de police d'Angers.

12. Cette suppression était déjà demandée par Charles Colbert dans le rapport qu'il adressait au roi en 1664 .

13. Arch. dép. de Maine-et-Loire, 1 B 2, f ${ }^{\circ} 142$. Les officiers du présidial déclarent que l'institution est chargée de 50000 livres de dettes. 
sommes nécessaires pour indemniser les officiers supprimés a été imposée sur les officiers de l'assemblée présidiale qui a dû également emprunter pour faire face à ses obligations. L'opération a coûté près de 100000 livres $^{14}$. En 1762, les dettes du tribunal angevin atteignent 150000 livres ${ }^{15}$. Dans ces conditions le présidial attire peu car chaque année, les nouveaux membres, comme les anciens, doivent payer leur part pour régler les intérêts des emprunts ce qui entraîne une diminution de leurs revenus ${ }^{16}$.

La diminution du nombre des membres du présidial et la forte perte de la valeur des offices de cette assemblée constituent le dernier aspect du déclin de l'institution judiciaire. Pendant de nombreuses années, le nombre des officiers du présidial, qui a varié à plusieurs reprises à la suite des créations d'offices par le pouvoir royal, a été de 39. En 1740, d'après le rapport adressé à l'intendant de la généralité de Tours par le subdélégué d'Angers, Poulain de la Guerche, conseiller au présidial, il y a encore 39 officiers dans le tribunal angevin ${ }^{17}$. Dans la seconde moitié du XVIII ${ }^{\mathrm{e}}$ siècle, ce nombre ne cesse de diminuer. Il est de 20 en $1772^{18}$ et de 17 en 1789 . Le sénéchal d'épée est toujours absent. Le 2 mars 1768, Augustin-Félix Barrin de la Galissonnière a été pourvu par le roi de la charge vacante par le décès de son frère ${ }^{19}$. Il a prêté le serment de fidélité au roi et a fait enregistrer ses lettres de provision par les cours souveraines parisiennes ${ }^{20}$ mais il ne procède aux formalités nécessaires à Angers que le 3 mars $1789^{21}$, afin de présider la séance solennelle d'ouverture de la réunion des députés des corps et communauté de la province pour les élections des députés aux États Généraux. Certes, le nombre des gens du roi n'a pas diminué : il est toujours de quatre en $1789^{22}$, mais de nombreux offices ont été supprimés ou sont vacants. Alors qu'en 1699 les officiers supérieurs étaient au nombre de six, à la veille de la Révolution ils ne sont plus que deux en exercice, un lieutenant général criminel et un lieutenant particulier civil. Les deux offices

14. Bibliothèque municipale d'Angers, ms. 1123 (922), f ${ }^{\circ} 228$. Le 23 mars 1754, il faut emprunter 10000 livres pour rembourser l'office de lieutenant de la prévôté, Arch. dép. de Maine-et-Loire, 1 B 2, $\mathrm{f}^{\circ} 153$.

15. BnF, ms. Joly de Fleury $385, \mathrm{f}^{\circ} 68$.

16. Le conseiller Beaujouan, installé en 1740, est malade depuis 1741. Il est mis sous tutelle pour démence en 1750. Il doit payer sa part annuelle comme le montrent les comptes de curatelle qui le concernent. Il a payé " pour les dettes du présidial " 1808 livres de 1750 à 1771. Arch. dép. de Maine-et-Loire, E 1598.

17. Arch. dép. d'Indre-et-Loire, C 338. En y comprenant les gens du roi.

18. Bib. mun. d'Angers, ms. 1123 (922), mémoire des officiers du présidial au prince apanagiste.

19. Le 3 mai 1760. Celui-ci, pourvu de la charge le 4 mars 1757, n'avait pas fait enregistrer ses lettres de provisions à Angers.

20. Parlement, le 9 mai 1768, Chambre des Comptes, le premier juin 1768.

21. Arch. dép. de Maine-et-Loire, 1 B 195, f ${ }^{\circ}$ 150-151.

22. L'office de second avocat du roi, vacant depuis 1761 et supprimé en 1771 est rétabli en 1786 et celui de substitut du procureur du roi supprimé en 1771 est rétabli dès 1774 à la demande du prince apanagiste. En 1786 Brevet de Beaujour propose 4000 livres pour l'office d'avocat du roi évalué 13000 livres. Le conseil de Monsieur lui accorde une remise et il ne verse que 9000 livres aux parties casuelles, Arch. nat., R5/24, p. 488. 
de président ont été supprimés par l'arrêt du conseil du 18 février $1755^{23}$ L'office de lieutenant particulier criminel est vacant depuis le décès de Frain du Tremblay en 1777, et celui de lieutenant général civil depuis la mort de Marcombe en $1780^{24}$. L'office de lieutenant particulier dont le prix avait été évalué à 12000 livres en 1772 , n'est vendu que 8000 livres en $1788^{25}$. Quant au nombre des conseillers qui atteignait 28 en 1740 d'après le subdélégué, il a été considérablement réduit ${ }^{26}$. En juin 1771 , un édit royal a décidé de supprimer les offices vacants " et dont la conservation n'apparaît pas nécessaire " et n'a conservé que les offices de douze conseillers " actuellement remplis ${ }^{27}$ ". Mais très vite ce nombre n'est plus atteint. En 1780, sept conseillers seulement sont en état d'assurer leurs fonctions. Deux offices sont en effet vacants, un autre est possédé par Jean-Pierre Beaujouan interdit pour "démence et dérangement d'esprit " et, depuis 1750, sa famille essaie en vain de vendre cet office inutile ${ }^{28}$. Enfin, deux conseillers ont quitté Angers et sont partis chercher fortune à Saint-Domingue ${ }^{29}$. Cette forte diminution gêne le fonctionnement de la justice car parmi les conseillers nombreux sont ceux qui sont âgés. En 1775, François Prévost, avocat du roi écrit au garde des sceaux que plusieurs conseillers sont " des vieillards et des infirmes " et qu'ils ne peuvent suivre avec assiduité les séances ${ }^{30}$, et cette situation " occasionne à ceux qui la composent un travail ardu et continu $^{31}$ " déclarent les officiers de la compagnie. Des offices sont vacants parce que leurs possesseurs essaient en vain de les vendre, malgré l'im-

23. Arch. nat., E 2340. Cet arrêt les unit à perpétuité à ceux de lieutenant général civil et de lieutenant général criminel. L'office de premier président vacant depuis le décès de Crespy en 1753 disparaît alors, celui de second président, possédé par Pays du Vau, ne doit disparaître qu'après le remboursement du prix de son office par les lieutenants généraux. Depuis 1759 il n'apparaît plus dans les assemblées du présidial. L'office a-t-il été vendu avant l'édit de 1764 portant suppression des offices des présidents dans tous les bailliages et présidiaux? D’après un mémoire des officiers du présidial, daté du 28 avril 1764, les deux offices de président ont été supprimés et réunis aux offices des lieutenants généraux, Bib. mun. d'Angers, C 19547.

24. Arch. dép. de Maine-et-Loire, 2 E 2168. Pays du Vau avait acheté cette charge pour son fils mineur, mais celui-ci décède, le 28 août 1783, avant d'avoir reçu ses lettres de provisions.

25. Arch. dép. de Maine-et-Loire, C 594. Vente par Gaspard Ollivier à Marie Milscent, le 5 janvier 1788 .

26. La liste des conseillers établie en 1742 par François Prévost, avocat du roi, comporte encore 26 noms, Bib. mun. d'Angers, ms. 1027 (895). En 1764, dix offices sont vacants et quatre ne sont pas exercés : il y a deux conseillers à Saint-Domingue, un est " dément "et un autre est infirme et ne participe plus aux séances.

27. Arch. dép. de Maine-et-Loire, 1 B 39.

28. En juillet 1768, la famille essaie de vendre la charge " au plus offrant et dernier enchérisseur " après avoir multiplié les annonces aux prônes et aux carrefours de la ville, personne ne se présente chez le notaire Deville Arch. dép. de Maine et Loire, 5 E 1/ 1074. L'office n'est vendu qu'en 1784, après le décès de Beaujouan, Arch. dép. de Maine-et-Loire, 5 E $5 / 201$ et 1 B $193, f^{\circ} 250$.

29. Le conseiller Davy en 1763, et Margariteau en 1769. Ils deviennent tous les deux conseillers au Conseil supérieur du Cap.

30. Bib. mun. d'Angers, ms. 1123 (922), f 210.

31. Arch. dép. de Maine-et-Loire, 1 B 2, f ${ }^{\circ} 182$. 
portante diminution de leur prix. En juillet 1740, le subdélégué évoquait cette situation en écrivant à l'intendant « il est certain que les charges de judicature ont tombés dans cette ville dans un grand discrédit. Aujourd'hui on a pour 6000 et 7000 livres une charge de conseiller en la sénéchaussée et siège présidial qui se vendait il y a 20 et 25 ans 18000 à 20000 livres ". En 1772, tous les conseillers ont évalué le prix de leur office à 8500 livres. Mais les membres du conseil du prince apanagiste considèrent que cette évaluation est " follement arbitraire ${ }^{32}$ ". En effet, la réalité est différente. Les offices se vendent mal et leur prix ne cesse de diminuer. Margariteau accusé d'avoir possédé à la fois une charge de conseiller au conseil supérieur du Cap à Saint Domingue et sa charge angevine se défend en affirmant " je n'ai pu vendre [...] à cause du discrédit des charges qui se vendent $\mathrm{mal}^{33}$ ". En 1770, Gabriel Maussion vend 3740 livres une charge achetée 7000 livres en $1732^{34}$. En 1775, Béguyer fait l'acquisition pour 5000 livres de l'office de Lebreton et, en 1783, René Jean-Baptiste-Louis de La Revellière ne verse que 1800 livres pour l'office que lui cède Verdier de la Miltière ${ }^{35}$. Certes, dans les années qui précèdent la Révolution les prix des offices augmentent à nouveau, et le nombre des offices pourvus remonte à onze ${ }^{36}$, mais cette augmentation est faible car les charges ne sont vendues que $2000^{37}$ ou 2400 livres seulement ${ }^{38}$. Les offices de procureur créés en 1772 lors de la désunion des fonctions d'avocats et de procureurs se négocient alors à des sommes bien supérieures : entre 8000 et 10000 livres ${ }^{39}$. L'amertume des officiers du présidial est grande, comme ils l'écrivent au prince apanagiste, en lui demandant, en vain, de faire accorder de nouveaux privilèges aux magistrats de la capitale de son apanage : "Le dégoût universel pour

32. Arch. nat., $\mathrm{R}^{5} / 26$, p. 130 . Pensant que le roi allait supprimer les offices, les titulaires en auraient surévalué le prix.

33. Arch. dép. de Maine-et-Loire, 2 E/1922.

34. Arch. dép. de Maine-et-Loire, 5 E 1/1776.

35. Mais Verdier de la Miltière n'avait pas payé le centième denier. Le mémoire des sommes que La Revellière doit verser à Monsieur (991 livres), au roi (1575 livres), pour sa réception au parlement (442 livres) et pour l'enregistrement de ses lettres de provision à la Chambre des comptes, au Bureau des finances de Tours et à Angers, montre que l'office lui revient au total à 5657 livres, (Arch. dép. de Maine-et-Loire, E 3773).

36. Il n'y a cependant que dix conseillers en exercice car Margariteau, rentré de SaintDomingue en 1783, ne participe pas aux travaux de la compagnie.

37. Arch. dép. de Maine-et-Loire, 5 E 7/644. Revenu de Saint-Domingue, Davy vend son office à Joseph Bariller, le 14 mai 1787 pour 2000 livres,

38. Arch. nat., R $/ 26$, p. 130. En 1787, Gilly demande à relever l'office vacant aux parties casuelles de Monsieur, depuis le décès de son père en 1782 . Il propose 2400 livres au lieu des 8500 livres de l'évaluation de 1772. En " considération du discrédit universel des offices de judicature " le conseil de Monsieur accepte une diminution des sommes à verser en évaluant l'office à 4250 livres.

39. Ainsi, le 29 décembre 1787, Jean Monteau vend son office pour 9300 livres : 1000 livres pour « le titre de l'office " et 8300 livres pour les pratiques et la clientèle, Arch. dép. de Maine-et-Loire, 5 E 6/ 371, notaire Macé. En janvier 1784, alors que Couraudin de la Noue achète pour 3000 livres l'office du conseiller Beaujouan, Joseph Morna, juge des traites et impositions foraines d'Anjou, vend son office 4000 livres, Arch. dép. Maine-etLoire 5 E 5/201. 
les offices de judicature procède du défaut d'autorité dans le magistrat et de ce que ses fonctions pénibles et continuelles ne présentent qu'une perspective de travaux rebutants qui ne se trouvent balancées par aucune récompense ni privilèges, pour le magistrat ni pour sa postérité ${ }^{40}$. " Dans ces conditions quels sont les hommes et les familles qui font l'acquisition de telles charges?

\section{Des hommes nouveaux}

" On offre son office, on le fait publier et afficher : il n'est pas un jeune homme de famille qui daigne l'accepter, même gratuitement [...]. Nos enfants mêmes refusent de nous remplacer, telle est l'opinion du jour qu'ils murmurent d'avoir pour pères des magistrats et qu'ils croiraient s'avilir de nous succéder ${ }^{41}$."

Cette plainte des officiers du présidial d'Angers, dans un mémoire adressé au roi en 1769 , traduit bien la réalité. Le présidial n'attire plus les enfants des vieilles et riches familles angevines ${ }^{42}$. On assiste à la fin de la présence des grandes dynasties familiales en son sein. Avant 1750, les offices se transmettaient de père en fils, ou d'oncle à neveu et de beaupère à gendre. En 1693, Pocquet de Livonnière déclarait qu'à Angers, on ne vendait pas les offices du présidial dans le commerce et que depuis des années personne n'en avait acheté car " les changements arrivés sont accommodements de famille ${ }^{43}$ ". Après 1750 , la situation est différente. Le symbole de cette évolution est, en 1774, la fin de la présence des Ayrault de Saint-Henis, qui depuis 1568 possédaient la charge de Lieutenant général criminel $^{44}$. Durant la période que nous étudions nous ne rencontrons que deux résignations familiales : en 1752, Pierre Ayrault succède à son père dans la charge de Lieutenant général criminel et en 1758, René Berthelot résigne son office en faveur de son demi-frère Joseph ${ }^{45}$. Après 1758 , il n'y a aucune résignation familiale et, en 1789, Joseph Berthelot est le seul des officiers alors en exercice à avoir succédé à un membre de sa famille. Les autres sont des hommes nouveaux dont la famille est entrée avec eux au

40. Bib. mun. d'Angers, ms. 1123 (922), f 228.

41. Bib. mun. d'Angers, C 19546, Mémoire au Roi. Des mémoires identiques avaient été adressés au roi par les officiers du présidial en 1752, 1763 et 1764.

42. Celles-ci sont alors nombreuses au sein de la Chambre des comptes de Bretagne, à Nantes, dont les offices procurent la noblesse. Sur les 166 officiers qui ont intégré la cour souveraine nantaise de 1750 à 1789, 43 sont des Angevins, soit $26 \%$ de l'ensemble, d'après JARNouX, Philippe, «D'Anjou en Bretagne : mobilités géographiques et mobilités sociales dans la France du XVIII ${ }^{\mathrm{e}}$ siècle ", dans HAudrère, Philippe (dir.), Pour une histoire sociale des villes. Mélanges offerts à Jacques Maillard, Rennes, 2006, p. 205-218.

43. Arch. dép. de Maine-et-Loire, 1 B 1.

44. La charge est vendue 30000 livres, alors qu'elle avait été évaluée à 43500 livres en 1772. Le prix de vente ne prend pas en compte 6000 livres versées pour l'achat des offices de la prévôté et 7500 livres pour ceux des deux offices de présidents supprimés, Arch. dép. de Maine-et-Loire, 5 E 2/425, notaire Bardoul.

45. La charge avait été occupée par leur père de 1714 à 1740. 
sein de l'assemblée judiciaire angevine. Comme l'écrivait, dès 1740, le subdélégué Poulain de la Guerche, le prix modique des charges " les met à portée d'être achetées par tout le monde. Ceux dont la naissance avait quelque chose au dessus des autres, dont les pères et ayeuls étaient dans le présidial, ont vu avec quelque peine recevoir des gens un peu nouveaux, ils se sont dégoûtés".

Une telle évolution se lit dans l'origine sociale des officiers ${ }^{46}$. On assiste, tout d'abord, à la disparition de la noblesse de robe. Après 1758, aucun des nouveaux officiers n'est noble et, en 1789, seul Joseph Berthelot appartient au second ordre. La majorité de ceux qui sont entrés après 1750 appartient toujours au monde des officiers royaux, soit $40 \%$, mais les fils d'officiers au présidial ne sont plus de $12 \%$ des nouveaux officiers. Dans les années qui précèdent la Révolution, 30 \% ont pour parents des officiers royaux ou seigneuriaux des petites villes de la province comme Vihiers ou Montaigu ${ }^{47}$. On assiste aussi à la montée des fils d'avocats qui représentent $40 \%$ des officiers de la compagnie et à celle des fils de bourgeois et de marchands ${ }^{48}$. Ces hommes nouveaux sont des hommes jeunes : cinq des six officiers supérieurs ont été reçus avant d'avoir les trente années requises et $30 \%$ des conseillers rapporteurs avant d'avoir atteint l'âge de 25 ans $^{49}$. Ils ont tous obtenu une lettre de dispense d'âge. L'entrée au présidial marque le début d'une carrière judiciaire pour des hommes n'ayant jamais exercé dans un tribunal royal auparavant ${ }^{50}$. En outre, $8 \%$ de ces juges sont célibataires lors de leur réception. Ils ont reçu presque tous la même formation puisque plus de $90 \%$ d'entre eux ont été étudiants à la faculté des droits de l'université d'Angers ${ }^{51}$. Tous se sont fait recevoir comme avocats au parlement de Paris, comme nous l'indiquent leurs lettres de provisions et $36 \%$ ont également été immatriculés à Angers, sans avoir, pour la plupart, exercé la profession d'avocat.

Ces nouveaux venus, qui commencent leur carrière judiciaire dans les années qui précèdent la Révolution, sont moins riches que ceux qui les ont précédés ${ }^{52}$. En l'absence de documents en nombre suffisant, il est difficile de présenter leur fortune, mais quelques faits nous paraissent significatifs.

46. Cette étude est réalisée pour les 25 officiers reçu au sein du présidial de 1750 à 1790 : six gens du roi, six officiers supérieurs et treize conseillers rapporteurs.

47. Le père de Desmazières était sénéchal de Vihiers, celui de Boyleau contrôleur au grenier à sel de Vihiers et celui de La Revellière juge des traites de Montaigu.

48. Le père de Béguyer était avocat, mais son grand'père était propriétaire d'une des raffineries de sucre d'Angers.

49. Ainsi, le 28 janvier 1784, Aimé Couraudin de la Noue achète pour 3000 livres l'office de Beaujouan. Il vient d'avoir 21 ans.

50. Certains, comme La Revellière, ont toutefois été sénéchal d'une justice seigneuriale.

51. Nous pouvons retrouver leurs noms sur les cahiers d'appel des étudiants, Arch. dép. de Maine-et-Loire, 3 D 1 à 3D 3 et 1 B 153. Parmi eux $20 \%$ ont obtenu leur doctorat en droit à Angers.

52. Louis Ayrault, entré au présidial en 1737 et qui emploie 3 domestiques depuis 1765 a une fortune modeste, lui aussi. En 1787, à cause de la médiocrité de ses ressources, Monsieur lui accorde une gratification de 1700 livres, Arch. nat., R R/24, p. 160. 
Ils n'habitent plus dans de grands hôtels, mais ils possèdent ou louent des demeures plus modestes, qui sont toutefois toujours situées, comme celles de leurs prédécesseurs, sur la rive gauche de la Maine, dans les rues et les paroisses où vivent les plus riches habitants d'Angers. Le nombre de leurs domestiques a diminué, comparé à celui des officiers du début du siècle. D'après les registres de la capitation qui nous permettent de les compter ${ }^{53}$, les officiers du présidial employaient en moyenne 2,8 domestiques en 1750, ils n'en ont plus que 2,2 seulement en 1789 , les officiers supérieurs ayant, à cette date, plus de trois domestiques et les juges ordinaires au moins deux $^{54}$. Les juges qui viennent d'entrer au présidial sont plus jeunes, ont moins d'enfants que leurs prédécesseurs, leur maison nécessite moins de domestiques. Cette diminution ne doit pas faire oublier toutefois que par leur nombre de domestiques les officiers du présidial apparaissent toujours en 1789 comme des privilégiés dans une ville où seule une petite minorité emploie deux domestiques.

En 1789, le présidial reste le principal tribunal d'Angers et de l'Anjou. Ses membres participent en corps à toutes les cérémonies publiques, en veillant scrupuleusement au respect de tous leurs droits, au respect de toutes leurs préséances, symboles de leur place dans la vie de la cité. Mais les membres du tribunal n'appartiennent plus aux grandes familles de la ville et de la province. Dans les années qui précèdent la Révolution, des hommes nouveaux, jeunes pour la plupart y commencent leur carrière. Auraient-ils donné naissance à de nouvelles grandes familles judiciaires? La disparition du tribunal à la fin de l'année 1790 ne leur permet pas de créer de nouvelles dynasties ${ }^{55}$.

53. Ces registres sont conservés aux archives municipales d'Angers pour les années 1712-1789. Chaque année ceux qui ont des domestiques paient trois ou quatre livres par domestique.

54. Parmi les juges élus après 1770, aucun n'a plus de trois domestiques.

55. Quelques-uns de ces hommes nouveaux ont exercé des fonctions dans les tribunaux angevins de la Révolution. Ainsi, Aimé Couraudin de la Noue est président du tribunal du district d'Angers de novembre 1792 à septembre, avant d'être exécuté en avril 1794. 


\section{RÉSUMÉ}

Dans les années qui précèdent la Révolution, le présidial d'Angers est en déclin. Ses membres ne dominent plus les principales institutions de la ville. Les grandes familles ne siègent plus dans un tribunal dont le prix des offices ne cesse de diminuer. Des offices vacants sont supprimés. Des hommes nouveaux, moins fortunés que les anciens, jeunes pour la plupart, souvent célibataires, commencent leur carrière dans ce tribunal dont l'accès leur a été longtemps fermé.

\section{ABSTRACT}

In the years leading up to the Revolution, the présidial of Angers was in decline. Its members no longer dominated the town's main institutions. The most important families no longer sat in a tribunal with offices of continually decreasing value. Vacant offices were suppressed. New men, less wealthy than their predecessors, young for the most part, and often single, started their careers in this tribunal which had previously been closed to them. 